Editorial

\title{
Plans for a New Online Open Access Journal: VCOT Open
}

Kenneth A. Johnson ${ }^{1}$

\author{
${ }^{1}$ Sydney School of Veterinary Science, University of Sydney, Sydney, \\ New South Wales, Australia \\ Vet Comp Orthop Traumatol 2018;31:v.
}

An exciting initiative planned for 2018 is the creation of a brand-new completely online journal to be called 'VCOT Open'. Designed primarily for the publication of case reports and reviews, this new journal will exist solely as an online journal, providing free access of articles to all readers.

In all other respects, the scope, instructions for authors and peer-review process of VCOT Open will be quite similar to Veterinary and Comparative Orthopaedics and Traumatology (VCOT), except that the publication of case reports in VCOT will be phased out.

VCOT will, of course, continue to be a subscription-based journal with a 'hybrid' format; authors who publish in VCOT can also elect to make their article freely available as an open access article, with the payment of an open access fee to our

publishers. Further details about VCOT Open will be forthcoming.

We wish all our readers, authors and review board members all the best for the coming year.

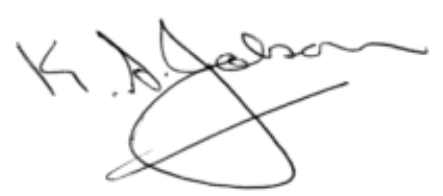

Kenneth A. Johnson

Editor-in-Chief

Sydney, Australia

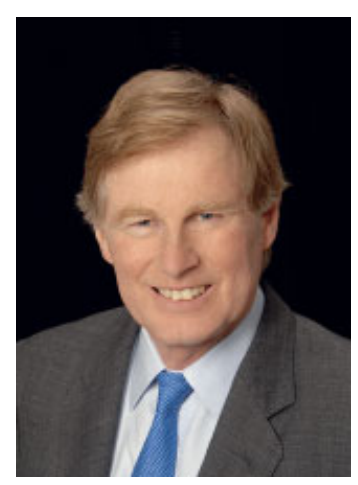

Kenneth A. Johnson 